\title{
Aneuploidy impairs hematopoietic stem cell fitness and is selected against in regenerating tissues in vivo
}

\author{
Sarah J. Pfau, ${ }^{1,2,3}$ Rebecca E. Silberman, ${ }^{1,2,3}$ Kristin A. Knouse, ${ }^{1,2,3,4}$ and Angelika Amon ${ }^{1,2,3}$ \\ ${ }^{1}$ David H. Koch Institute for Integrative Cancer Research, Massachusetts Institute of Technology, Cambridge, Massachusetts \\ 02139, USA; ${ }^{2}$ Howard Hughes Medical Institute, Massachusetts Institute of Technology, Cambridge, Massachusetts 02139, USA; \\ ${ }^{3}$ Department of Biology, Massachusetts Institute of Technology, Cambridge, Massachusetts 02139, USA; ${ }^{4}$ Division of Health \\ Sciences and Technology, Harvard Medical School, Boston, Massachusetts 02115, USA
}

\begin{abstract}
Aneuploidy, an imbalanced karyotype, is a widely observed feature of cancer cells that has long been hypothesized to promote tumorigenesis. Here we evaluate the fitness of cells with constitutional trisomy or chromosomal instability (CIN) in vivo using hematopoietic reconstitution experiments. We did not observe cancer but instead found that aneuploid hematopoietic stem cells (HSCs) exhibit decreased fitness. This reduced fitness is due at least in part to the decreased proliferative potential of aneuploid hematopoietic cells. Analyses of mice with CIN caused by a hypomorphic mutation in the gene Bub1b further support the finding that aneuploidy impairs cell proliferation in vivo. Whereas nonregenerating adult tissues are highly aneuploid in these mice, HSCs and other regenerative adult tissues are largely euploid. These findings indicate that, in vivo, mechanisms exist to select against aneuploid cells.
\end{abstract}

[Keywords: aneuploidy; chromosomal instability; hematopoiesis; single-cell sequencing; population flush hypothesis]

Supplemental material is available for this article.

Received February 1, 2016; accepted in revised form May 27, 2016.

More than $90 \%$ of human solid tumors are aneuploid (Rajagopalan and Lengauer 2004). It was initially hypothesized in 1914 that aneuploidy may play a causal role in tumorigenesis (Boveri 1914), and recent analyses of tumor genomes support this idea, finding that aneuploidies observed in cancer can be explained in part by loss of tumor suppressor genes and gain of oncogenes (Davoli et al. 2013). Despite the prevalence of imbalanced karyotypes in cancer, in vitro studies of aneuploid cells have not provided decisive evidence that aneuploidy alone can promote tumorigenesis. Trisomic human (Segal and McCoy 1974; Stingele et al. 2012) and mouse (Williams et al. 2008) cells exhibit decreased proliferation, and cells harboring mutations that promote chromosomal instability (CIN) proliferate either normally (Babu et al. 2003; Jeganathan et al. 2007; Weaver et al. 2007) or more slowly, indicating that aneuploidy is, at best, fitness-neutral. Furthermore, studies in mouse models of CIN and Down syndrome (DS) have demonstrated that these conditions can both promote and inhibit tumorigenesis in vivo (Pfau and Amon 2012). These observations make clear the need for additional experimental systems to assess the effects of aneuploidy per se on cell proliferation in vivo. However, systematic evaluation of aneuploid cell fitness

Corresponding author: angelika@mit.edu

Article published online ahead of print. Article and publication date are online at http://www.genesdev.org/cgi/doi/10.1101/gad.278820.116. in vivo is difficult because most autosomal aneuploidies are embryonic-lethal in mammals.

To bypass the embryonic lethality of aneuploidy in mice, we performed transplantation experiments with hematopoietic stem cells (HSCs) isolated from mouse embryos. HSCs provide a cellular system that is amenable for systematic study of the effects of aneuploidy in vivo because HSCs give rise to all differentiated blood cell types (Kondo et al. 2003). Whereas HSCs reside in the bone marrow of adult mice, they can be found in the fetal liver between embryonic day 12.5 (E12.5) and E15.5 (Orkin and Zon 2008), allowing the isolation of HSCs from fetal livers of aneuploid embryos that survive to this embryonic age.

High doses of irradiation ablate the hematopoietic function of HSCs; however, transplantation of HSCs from a nonirradiated donor can reconstitute the hematopoietic system of an irradiated recipient. Thus, aneuploid fetal liver HSCs (FL-HSCs) can be used to reconstitute the blood of a lethally irradiated wild-type recipient, giving rise to an otherwise wild-type mouse with aneuploid blood. Hematopoietic reconstitutions performed with

(C) 2016 Pfau et al. This article is distributed exclusively by Cold Spring Harbor Laboratory Press for the first six months after the full-issue publication date (see http://genesdev.cshlp.org/site/misc/terms.xhtml). After six months, it is available under a Creative Commons License (Attribution-NonCommercial 4.0 International), as described at http://creativecommons.org/licenses/by-nc/4.0/. 
FL-HSCs derived from euploid wild-type littermates allow for direct comparison of aneuploid and euploid HSC fitness. Thus, the hematopoietic compartment represents an ideal in vivo system to assess the fitness of aneuploid karyotypes that cause embryonic lethality. Analyzing HSC fitness in vivo not only permits evaluation of aneuploid cell proliferative capacity but also provides insight into how aneuploidy affects stem cell potential, as previous studies have demonstrated that aneuploidy can perturb stem cell viability and differentiation (Adorno et al. 2013; Gogendeau et al. 2015). Finally, this system also offers a unique model for studying aneuploid cells in the context of an otherwise euploid environment, a setting typical of tumorigenesis in vivo.

We chose three mouse models of aneuploidy to evaluate the fitness of aneuploid HSCs: constitutional trisomy 16, constitutional trisomy 19 , and mice harboring a hypomorphic allele of the gene $B u b 1 b$, which encodes the spindle assembly checkpoint protein BUBR1 $\left(B u b 1 b^{H / H}\right)$ (Baker et al. 2004). Mouse chromosome 16 is the closest wholechromosome homolog to human chromosome 21 and thus a model of DS. This model is of particular interest because individuals with DS often show perturbations in the hematopoietic lineage (Henry et al. 2007; Choi 2008) and have a greatly increased risk of developing childhood leukemia (Satge et al. 1998). Mouse chromosome 19 is the smallest mouse autosome, with homology mainly with human chromosomes 9, 10, and 11. While mouse chromosome 19 is not known to harbor genes typically associated with acute myeloid leukemia (AML), activating mutations in JAK2 (encoded by mouse chromosome 19) are frequently associated with myeloproliferative neoplasms (Kiladjian 2012). The Bub1b ${ }^{H / H}$ CIN model produces aneuploid cells with mostly single-chromosome gains or losses, thus representing a variety of aneuploid chromosomes due to random missegregation events (Baker et al. 2004). Bub1b $b^{H / H}$ mice survive to adulthood, permitting comparison of both fetal liver and adult bone marrow $B u b 1 b^{H / H}$ HSCs with constitutional trisomic FL-HSCs. $B u b 1 b^{H / H}$ mice develop progeria-like symptoms and have a decreased life span but do not develop cancer (Baker et al. 2004).

A comparison of HSCs from these three models has revealed a range of responses to aneuploidy in the blood and permitted differentiation between chromosome-specific and general effects of aneuploidy in vivo. We found that while some aneuploidies can be well tolerated in the hematopoietic lineage, aneuploidy generally causes a decrease in HSC fitness. This decreased fitness is at least partially due to the decreased proliferative potential of aneuploid hematopoietic cells. Additional analyses of $B u b 1 b^{H / H}$ CIN mice show that aneuploidy is tolerated in this strain during periods of rapid hematopoietic population expansion. However, single-cell sequencing of tissues from adult $B u b 1 b^{H / H}$ mice revealed that aneuploidy is not uniformly tolerated across different adult tissue types. While tissues that are largely nonproliferative in the adult display high levels of aneuploidy, regenerative tissues harbor few, if any, aneuploid cells. These data provide evidence that aneuploidy-selective mechanisms eliminate aneuploid cells during adult hematopoiesis and likely in other tissues that regenerate during adulthood.

\section{Results}

\section{Aneuploidy decreases HSC competitive fitness in vivo}

To determine the effect of aneuploidy on cell fitness in vivo, we first used competitive reconstitution assays to evaluate the fitness of aneuploid FL-HSCs. In this assay, two populations of HSCs were coinjected into a lethally irradiated recipient, and the relative contributions of each population to the hematopoietic compartment were evaluated over time by analysis of the peripheral blood. To ensure that equal numbers of cells were being competed, we first measured HSC levels. Quantification by flow cytometry revealed no significant differences in the HSC levels in trisomy 16 or trisomy 19 fetal livers (Fig. 1A). Because Bub1b $b^{H / H}$ animals are viable, we quantified HSC levels in the adult and found them to be similar to those of their wild-type littermates (Supplemental Fig. S5I). Thus, we concluded that HSC levels are similar in aneuploid and euploid donors.

To assess the fitness of aneuploid HSCs, we injected equal numbers of live aneuploid or euploid littermate control fetal liver cells into a lethally irradiated euploid recipient together with the same number of live fetal liver cells from a common euploid competitor of the same embryonic age (referred to here as common wild type) (Fig. 1B). To distinguish between experimental HSCs and the common wild-type competitor, each donor was tracked using a different isoform of the pan-leukocyte cell surface marker CD45, which can be distinguished by isoform-specific antibodies (CD45.1 and CD45.2). Aneuploid donors and their wild-type littermates expressed the CD45.2 isoform, whereas the common wild-type competitor expressed the CD45.1 isoform. We chose to use a CD45.1 common donor because previous studies had shown that CD45.1 HSCs exhibit decreased fitness when compared with CD45.2 HSCs in competition assays (Waterstrat et al. 2010), thus giving the CD45.2 aneuploid donors a slight advantage in these experiments. Additionally, we used CD45.1 recipients to unambiguously quantify the contribution from aneuploid and euploid wild-type littermate donors. We further note that, in this experimental setup, a small population of recipient-derived memory $T$ cells remains in the recipient peripheral blood after reconstitution despite lethal irradiation (Frasca et al. 2000). This recipientderived memory cell population is evident in our data 3 wk after transfer (Fig. 1C-E). In these samples, an increased proportion of the blood is comprised of CD45.1-positive cells because donor-derived peripheral blood cells are present at low levels at this early stage of reconstitution, making the contribution from recipientderived memory cells more prominent.

Evaluation of competitive reconstitution assays over time revealed a range of aneuploid cell fitness phenotypes. Trisomy 16 FL-HSCs were much less fit than FL-HSCs from their wild-type littermates and were nearly 
A

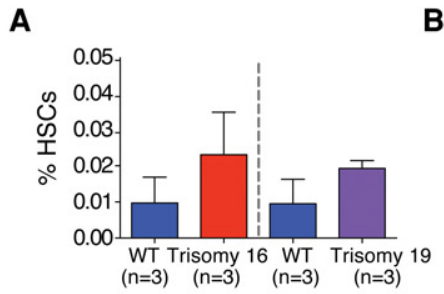

C

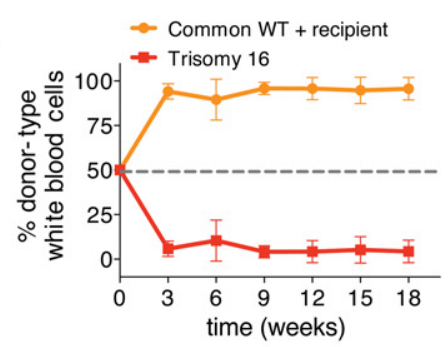

D

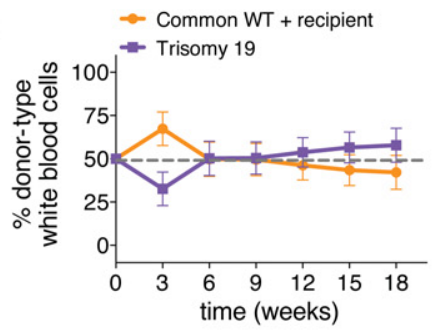

E

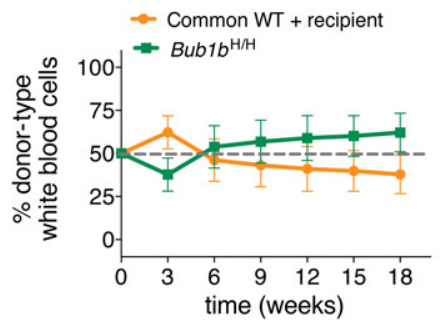

B $\quad$ CD 45.2

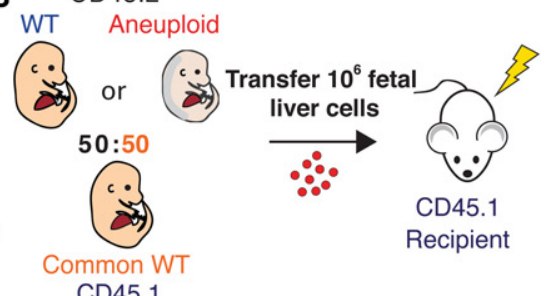

CD45.1
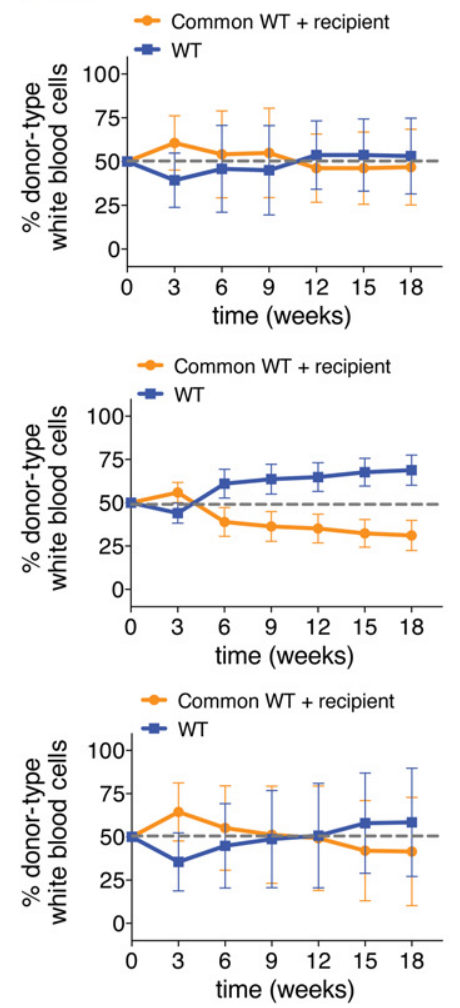

G
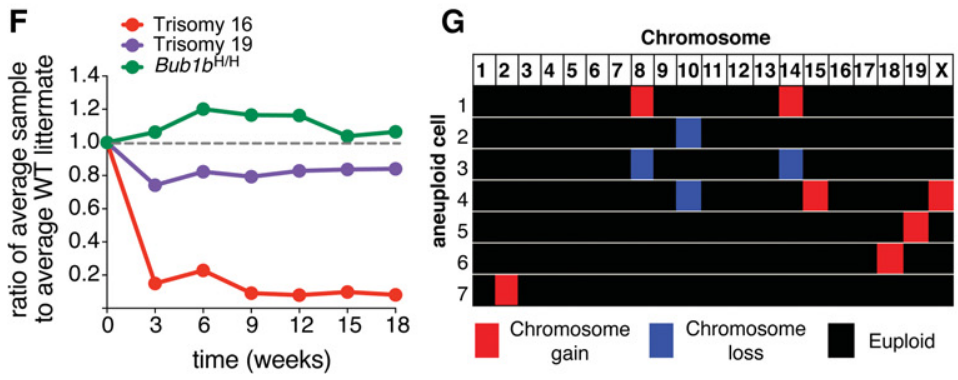

Figure 1. Aneuploidy decreases HSC competitive fitness in vivo. (A) The percentage of HSCs $\left(\mathrm{CD} 150^{+}\right.$ CD $48^{-}$Sca- $1^{+}$lin $^{-}$cells) found in trisomy 16 and trisomy 19 fetal livers was quantified by flow cytometry. Data are shown as mean \pm standard deviation. $(B)$ Schematic of competitive reconstitution experiments. (CE) CD45.2 fetal liver cells from wild-type or aneuploid E14.5 littermates were coinjected into a lethally irradiated CD45.1 recipient with an equal number of fetal liver cells from a CD45.1 common wild-type donor of the same age derived from a separate mating. Peripheral blood was sampled at the indicated times. The percentage of the white blood cell population contributed by each donor was quantified by flow cytometry with isoform-specific antibodies against CD45.1 and CD45.2 for recipients of common wild-type cells and trisomy 16 fetal liver cells $(C$, left graph) $(n=17)$, trisomy 19 fetal liver cells $\left(D\right.$, left graph) $(n=10)$, and $B u b 1 b^{H / H}$ fetal liver cells ( $E$, left graph) $(n=10)$. (C-E, right graphs) The contribution of wild-type littermates when competed to the common wild type for all aneuploidies was quantified at the same time in $C$ (right graph) $(n=20), D$ (right graph) $(n=8)$, and $E$ (right graph) $(n=6)$. Data are represented as mean \pm standard deviation for each time point. $(F)$ Ratios of the average percentage of the peripheral blood reconstituted by the aneuploid fetal liver cells to the average percentage of the peripheral blood reconstituted by wild-type littermate fetal liver cells at the indicated times are shown. $(G)$ Single-cell sequencing of white blood cells from a mouse competitively reconstituted with CD45.2 $B u b 1 b^{H / H}$ and CD45.1 euploid FL-HSCs at 16 wk after transplantation (Fig. 1E) revealed that seven of 18 CD45.2 Bub1 $b^{H / H}$ cells analyzed $(\sim 39 \%)$ were aneuploid. Karyotypes of the seven aneuploid cells are shown with chromosome gains in red, chromosome losses in blue, and euploidy in black. Segmentation plots of all sequenced cells are shown in Supplemental Figure S7A. completely outcompeted by the common wild-type donor (Fig. 1C,F). Trisomy 19 FL-HSCs exhibited slightly reduced relative fitness (Fig. 1D,F). $B u b 1 b^{H / H}$ FL-HSCs showed no relative fitness defects in this assay (Fig. 1E,F).

Because we did not observe decreased fitness in $B u b 1 b^{H / H}$ competitive reconstitutions, we assessed the level of aneuploidy in these CIN cells. We isolated peripheral white blood cells derived from $B u b 1 b^{H / H}$ FL-HSC donor cells from a recipient mouse $16 \mathrm{wk}$ after transfer. $B u b 1 b^{H / H}$ CD 45.2 cells were collected by FACS, and their karyotype was determined by single-cell sequencing. Analysis of 18 cells revealed seven to be aneuploid ( 38.9\%) (Fig. 1G; Supplemental Fig. S7A). This level of aneuploidy is on par with previously reported levels of aneuploidy for the brain $(38.1 \%)$ and the liver $(18.8 \%)$ (Knouse et al. 2014) as well as for stimulated splenocytes $(3 \%-33 \%)$ in adult Bub1 $b^{H / H}$ mice (Baker et al. 2004). Therefore, Bub1b $b^{H / H}$ FL-HSCs show fitness similar to that of euploid wild-type controls in this assay despite the prevalence of aneuploid cells in the peripheral blood.

In summary, when compared with the average fitness of euploid wild-type littermates after $18 \mathrm{wk}$, the average relative fitness of trisomy 16, trisomy 19 , and $B u b 1 b^{H / H}$ FL-HSCs was 0.08, 0.84, and 1.06, respectively (Fig. 1F). Thus, in this assay, some aneuploidies confer decreased HSC fitness, whereas others are fitness-neutral. These 
findings suggest either that the observed fitness decreases are due to chromosome-specific effects or that a certain level of aneuploidy is tolerated in the blood.

\section{Decreased fitness of aneuploid HSCs is due to decreased proliferation}

To determine why trisomy 16 and trisomy 19 FL-HSCs exhibited decreased relative fitness in competitive reconstitution assays, we first evaluated the ability of hematopoietic cells from each aneuploid donor to home to the bone marrow niche, a property that is essential to restore hematopoiesis during HSC transplantation (Lapidot 2005). Fetal liver cells were labeled with a fluorescent cell surface dye and injected into irradiated recipients. The number of labeled cells in the bone marrow was quantified after $24 \mathrm{~h}$. We observed no significant decrease in the homing efficiency of trisomy 16 , trisomy 19 , and $B u b 1 b^{H / H}$ fetal liver cells (Student's $t$-test) (Fig. 2A), suggesting that a defect in homing is not responsible for the decreased ability of aneuploid FL-HSCs to reconstitute the hematopoietic system of lethally irradiated recipients.

We next performed colony-forming unit spleen (CFU-S) assays to determine whether the proliferation of aneuploid FL-HSC-derived progenitor cells was impaired during early stages of hematopoietic reconstitution (Purton and Scadden 2007). Irradiated mice were injected with fetal liver cells, and spleens were analyzed 7 or $8 \mathrm{~d}$ later (Fig. 2B; Till and McCulloch 1961). Aneuploid and euploid fetal liver cells formed similar numbers of colonies in the spleen (Fig. 2C). However, quantification of the area of each CFU-S colony revealed that the average size of the colonies produced by trisomy 16 and trisomy 19 donor cells was reduced compared with colonies formed by cells from their wild-type littermates (Fig. 2D). The average size of $B u b 1 b^{H / H}$ CFU-S colonies was similar to those formed by wild-type littermate fetal liver cells (Fig. 2D).

To further evaluate the proliferative potential of aneuploid FL-HSCs, we measured EdU incorporation in the bone marrow of irradiated recipient mice reconstituted with trisomy 16 FL-HSCs. The number of EdU-positive donor-derived cells was significantly reduced compared with wild-type controls $6 \mathrm{~d}$ after reconstitution (Fig. 2E), indicating that trisomy 16 interferes with proliferation of FL-HSCs and progenitor cells. Consistent with this finding is the observation that trisomy 16 and trisomy 19 fetal livers were significantly smaller than fetal livers of their wild-type littermates (Supplemental Fig. S1). Interestingly, Bub1b $b^{H / H}$ fetal liver-derived cells did not exhibit decreased proliferation as evaluated by EdU incorporation $6 \mathrm{~d}$ after reconstitution; rather, there appeared to be a (not statistically significant) trend toward increased proliferation in these cells compared with wild-type control cells (Fig. 2E). The analyses of aneuploidy levels in $B u b 1 b^{H / H}$ tissues described below provide a potential explanation for this observation. Taken together, our data demonstrate that aneuploid FL-HSCs can home effectively but, in the cases of trisomies 16 and 19, show impaired proliferative potential.

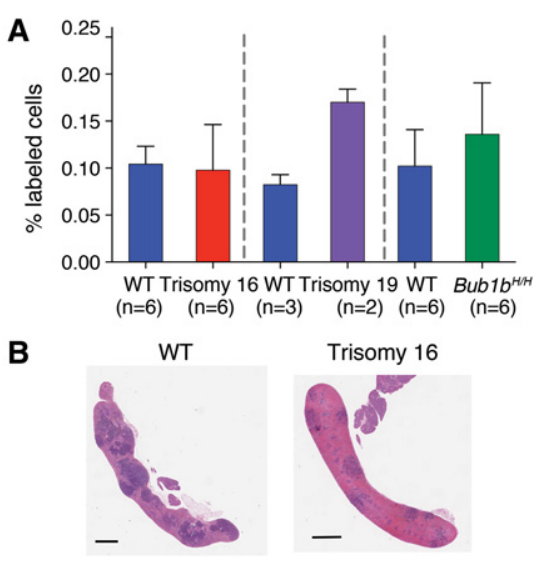

C
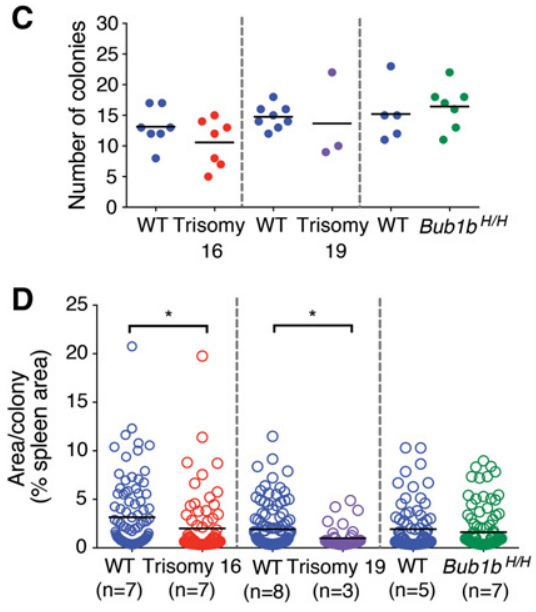

E

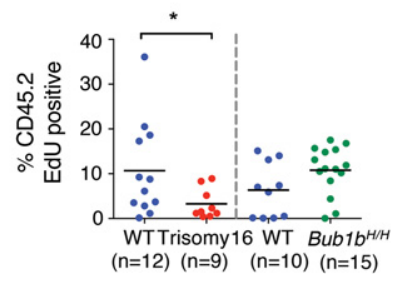

Figure 2. Proliferation but not homing ability is reduced in trisomy 16 and trisomy 19 reconstitutions. (A) DiI-labeled fetal liver cells were injected into irradiated recipient mice. The percentage of DiI-positive cells in the bone marrow of recipient mice was measured $24 \mathrm{~h}$ after injection. Data are shown as mean \pm standard deviation. (B) Representative images of sections of spleens isolated from mice transferred with wild-type or trisomy 16 fetal liver cells $8 \mathrm{~d}$ after reconstitution. Bar, $1 \mathrm{~mm}$. (C) Quantification of colony-forming unit spleen (CFU-S) colonies from spleen sections of recipient mice of trisomy 16 or wild-type littermate fetal liver cells $8 \mathrm{~d}$ after injection and trisomy $19, B u b 1 b^{H / H}$, or wild-type littermate fetal liver cells $7 \mathrm{~d}$ after injection. The bar represents the mean value for each condition. $(D)$ Quantification of the mean size of each colony in $C$ as determined by the percent of total spleen area. Measurements from all individuals for each condition were pooled. The bar represents the mean value for each population. Populations were compared by Student's $t$-test. $\left({ }^{*}\right) P<0.05$. $(E)$ Trisomy 16 or $B u b 1 b^{H / H}$ fetal liver cells or cells from their wildtype littermates were injected into lethally irradiated recipients. Mice were injected with EdU 6 d later, and the level of EdU incorporation in CD45.2-positive donor-derived bone marrow cells was evaluated by flow cytometry $24 \mathrm{~h}$ later. The bar represents the mean value for each population. $\left({ }^{*}\right) P<0.05$ by Student's $t$-test. 
Trisomy 16 recipients exhibit peripheral blood defects and decreased survival

Whereas trisomy 16 FL-HSCs exhibited a severe fitness defect in competitive reconstitution experiments, the fitness decrease was more subtle for trisomy 19 FL-HSCs and not evident for $B u b 1 b^{H / H}$ FL-HSCs. This suggests either that low levels of aneuploidy do not strongly impair HSC fitness or that HSCs were not challenged sufficiently in these assays to reveal decreased cellular fitness. To address the latter possibility, we evaluated the long-term fitness of HSCs from each aneuploid strain individually by serially transferring HSCs from primary to secondary and, in some cases, to tertiary and quaternary recipients (Fig. 3A). Serial transfer poses a significant challenge to the replicative potential of HSCs and their progeny, causing the eventual exhaustion of even wild-type HSCs (Harrison and Astle 1982). Trisomy 16 and trisomy 19 FL-HSCs have been evaluated previously in primary transplantation (Herbst and Winking 1991); however, these studies were not performed on an isogenic background, and it is therefore difficult to determine whether the observed phenotypes were due to aneuploidy or factors such as graft rejection (Gropp et al. 1983).
We transferred either CD45.2 trisomy 16 or CD45.2 euploid wild-type littermate fetal liver cells into lethally irradiated CD45.1 primary recipient mice (Fig. 3A). Peripheral blood analyses of primary recipients performed periodically between 4 and 16 wk after transfer revealed that trisomy 16 FL-HSCs harbor significant fitness defects when transferred individually (Fig. 3; Supplemental Fig. S2). These defects are consistent with decreased proliferation in the hematopoietic lineage. For example, trisomy 16 FL-HSCs contributed relatively fewer peripheral white blood cells than wild-type HSCs (Fig. 3B). Trisomy 16 primary recipients also exhibited leukopenia (reduced white blood cell counts) when compared with primary recipients reconstituted with cells from their euploid littermates (Fig. 3C). This seemed to be largely due to low numbers of B cells (Supplemental Fig. S2A,B,I). Furthermore, complete blood cell counts showed that trisomy 16 primary recipients have comparatively fewer red blood cells and macrocytic anemia, a reduced red blood cell count accompanied by increased red blood cell volume and decreased blood hemoglobin concentration (Fig. 3D; Supplemental Fig. S2C-G,J). These results are in line with previous in vivo characterizations of trisomy 16 FL-HSCs (Herbst and Winking 1991). In addition to

A
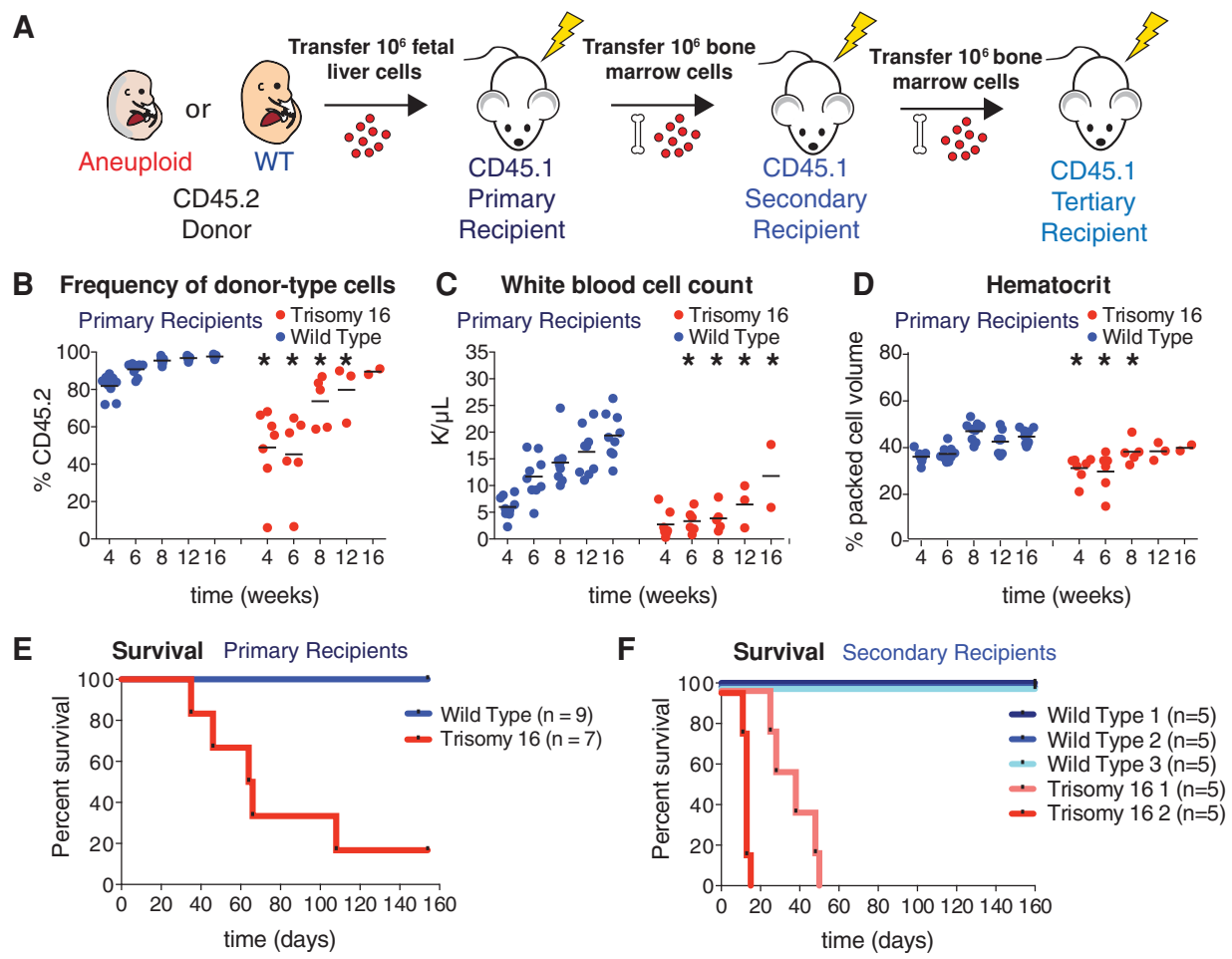

Figure 3. Trisomy 16 causes peripheral blood defects and decreases HSC reconstitution potential. (A) Schematic of serial reconstitution experiments. $(B-D)$ For primary reconstitutions, fetal liver cells from a CD45.2 trisomic embryo or its wild-type littermate were injected into lethally irradiated CD45.1 recipients. Bone marrow cells from primary recipients were injected into secondary CD45.1 recipients to assess serial reconstitution capacity. Peripheral blood of primary recipients of trisomic fetal liver cells or their wild-type littermates was sampled at the indicated times. The percentage of CD45.2-positive cells in the blood of trisomy 16 or wild-type primary recipients was determined by flow cytometry $(B)$, and white blood cell count $(C)$ and hematocrit $(D)$ were determined by complete blood cell counts. The bar represents the mean, and asterisks indicate that the trisomy 16 values are significantly different from the values of wild-type littermates at the indicated time by Student's $t$-test. $P<0.05$. (E) Survival of recipients of trisomy 16 or wild-type fetal liver cells after transfer. $(F)$ Survival of secondary recipients of trisomy 16 or wild-type bone marrow cells from primary recipients. 
peripheral blood defects, trisomy 16 primary recipients exhibited decreased survival compared with primary recipient mice reconstituted with HSCs from their euploid wild-type littermates (Fig. 3E).

As the ultimate test of HSC potential, secondary transfers were performed with bone marrow cells from trisomy 16 primary recipients that survived to $16 \mathrm{wk}$. Although long-term reconstituted primary recipients had comparable HSC levels in the bone marrow (Supplemental Fig. $\mathrm{S} 2 \mathrm{~K})$, transfer of wild-type primary recipient bone marrow cells efficiently reconstituted secondary recipients, while trisomy 16 primary recipient HSCs failed to reconstitute secondary recipients (Fig. 3F). We conclude that trisomy 16 FL-HSCs have a substantial fitness defect that is at least in part due to decreased proliferative potential, leading to HSC exhaustion upon secondary transfer.

\section{Trisomy 19 is better tolerated in the blood than} trisomy 16

In contrast to trisomy 16 FL-HSCs, trisomy 19 FL-HSCs contributed effectively to the white blood cell lineages in primary recipients (Fig. 4A; Supplemental Fig. S3A-J). Recipients reconstituted with trisomy 19 FL-HSCs showed normal white blood cell counts when compared with recipients reconstituted with wild-type littermate FL-HSCs (Fig. 4B), exhibited normal hematocrit levels (Fig. 4C), and did not show either red blood cell defects (Supplemental Fig. S3C-G) or macrocytic anemia (Supplemental Fig. S3J). However, trisomy 19 primary recipients harbored fewer platelets compared with wild-type primary recipients at early time points (Supplemental Fig. $\mathrm{S} 3 \mathrm{H})$. Furthermore, the proportion of the blood comprised of each white blood cell type in trisomy 19 primary recipients was not significantly perturbed (Supplemental Fig.
S3A,B,I). Consistent with trisomy 19 having little or no effect on HSC fitness in primary reconstitutions, we found that all mice transplanted with trisomy 19 HSCs survived both primary transfer of fetal liver cells and secondary transfer of bone marrow cells isolated from primary recipients (data not shown). Furthermore, secondary recipient mice showed no obvious peripheral blood defects (Supplemental Fig. S3K-U) except for a slightly weaker initial engraftment after 4 wk. However, this delay did not persist. In fact, the engraftment at 8 wk was stronger in the trisomy 19 HSC secondary recipients than in wild-type recipients (Student's two-tailed $t$-test, $P<0.05$ ) (Fig. 4D). Upon tertiary transfer, however, we began to see evidence of decreased fitness in some recipients of trisomy 19 bone marrow cells (Fig. 4E) even though HSC levels were similar in the bone marrow of trisomy 19 and wild-type long-term reconstituted secondary recipients (Supplemental Fig. S3V). While there were no obvious peripheral blood defects when compared with wild-type tertiary recipients (Supplemental Fig. S4A-K), weaker engraftment of trisomy 19 cells was evident in some tertiary recipients (Fig. 4E), and long-term survival of tertiary recipients was slightly decreased (Supplemental Fig. S4L). Trisomy 19 fitness defects became even more pronounced in quaternary transfers (Fig. 4F). This defect was not due to fewer HSCs being transferred. Trisomy 19 and wild-type long-term reconstituted tertiary recipients had comparable levels of HSCs (Supplemental Fig. S4M), but the majority of trisomy 19 quaternary recipient mice showed very low levels of engraftment compared with wild-type quaternary recipients (Fig. 4F). Lethality was high in both wild-type and trisomy 19 quaternary recipients (Supplemental Fig. S4O). Sequencing of the CD45.2 cells in two trisomy 19 quaternary recipients 14 wk after transfer confirmed that the trisomic
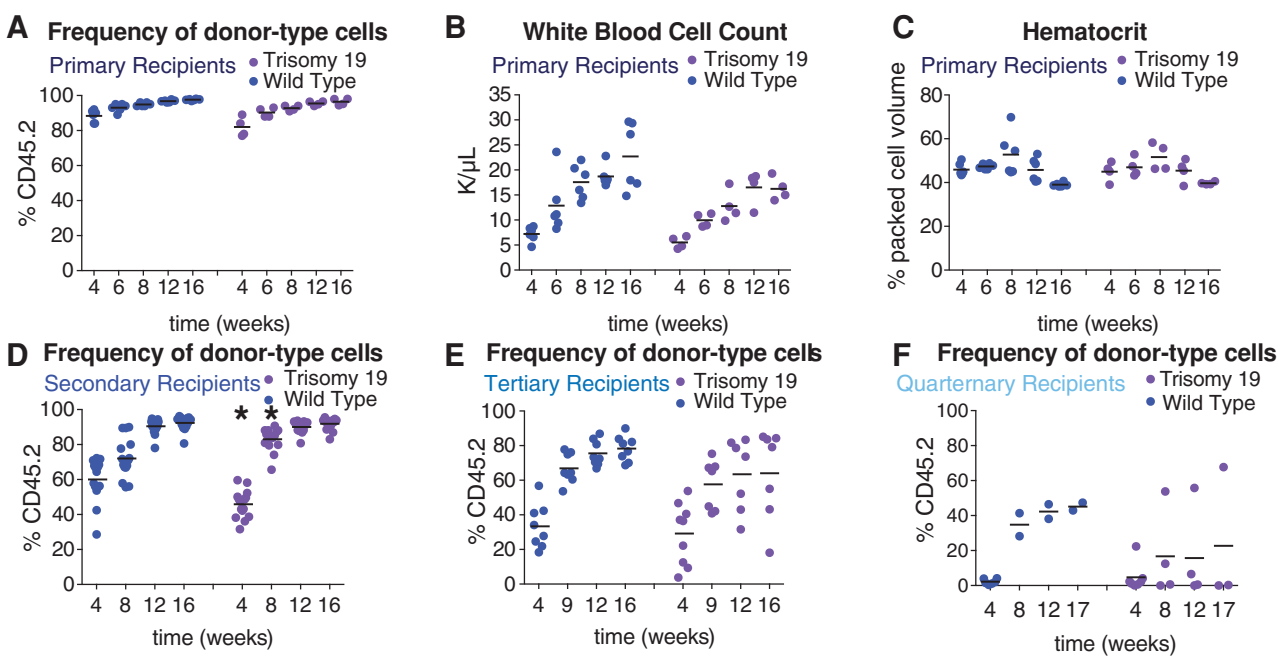

Figure 4. Trisomy 19 HSC reconstitution potential is reduced upon serial reconstitution. $(A-E)$ For trisomy 19 or wild-type primary recipients, the percentage of CD45.2-positive cells in the peripheral blood $(A)$, white blood cell count $(B)$, and hematocrit $(C)$ was determined. The percentage of CD45.2-positive cells in the blood of trisomy 19 or wild-type secondary recipients $(D)$, tertiary recipients $(E)$, and quaternary recipients $(F)$ was also evaluated. The bar represents the mean value for each condition, and asterisks indicate that the trisomy 19 values are significantly different from the values of wild-type littermates at the indicated time by Student's $t$-test. $P<0.05$. 
chromosome was retained through all serial transfers (Supplemental Fig. S4N). We conclude that trisomy 19 is better tolerated in the hematopoietic lineage than trisomy 16 , with no difference in survival and little difference in peripheral blood cell counts in both primary and secondary recipient mice. However, decreased reconstitution potential in tertiary and quaternary recipients indicates that aneuploidy of even the smallest mouse autosome will eventually lead to reduced fitness.

\section{Bub $1 b^{\mathrm{H} / \mathrm{H}}$ HSCs undergo stem cell exhaustion upon serial transplantation}

We next sought to evaluate the fitness of the $B u b 1 b^{H / H}$ CIN model, a model in which random combinations of whole chromosomal aneuploidies can be continuously generated by chromosome missegregation. To evaluate the long-term regenerative potential of $B u b 1 b^{H / H}$ HSCs, we used bone marrow HSCs (BM-HSCs) (Fig. 5A) because $B u b 1 b^{H / H}$ mice survive to adulthood (Baker et al. 2004) and show no obvious peripheral blood defects (Supplemental Fig. S5A-H). Like Bub1b $b^{H / H}$ FL-HSCs, Bub1b $b^{H / H}$ BM-HSCs showed no fitness defects in competitive reconstitution assays and were found at levels similar to their wild-type littermates (Supplemental Fig. S5I-L). As BMHSCs have already migrated from the fetal liver to populate the bone marrow of an adult mouse, we considered bone marrow transfer a more significant proliferative challenge than transfer of fetal liver cells. Also, FLHSCs have been shown to reconstitute irradiated recipients more effectively than BM-HSCs (Morrison et al. 1995; Harrison et al. 1997; Ema and Nakauchi 2000). However, Bub1b $b^{H / H}$ BM-HSCs contributed most of the peripheral white blood cells in primary recipient mice (Fig. 5B). The animals were mildly leukopenic but exhibited no other obvious blood defects (Supplemental Fig. S6A-I).
To further challenge $B u b 1 b^{H / H}$ HSCs, we performed serial bone marrow transfers (Fig. 5A). Despite similar total HSC levels in the bone marrow of long-term reconstituted $B u b 1 b^{H / H}$ and wild-type primary recipients (Supplemental Fig. S6J), bone marrow cells from primary $B u b 1 b^{H / H}$ recipients repopulated the hematopoietic compartment of secondary recipients less efficiently than wild-type primary recipient bone marrow cells (Fig. 5C). While the donor contributed most of the peripheral white blood cells in mice transferred with wild-type cells (on average, $87.3 \%$ at $12 \mathrm{wk} ; n=10$ ), variable contribution was observed in $B u b 1 b^{H / H}$ secondary recipients. Although complete blood cell counts of $B u b 1 b^{H / H}$ secondary recipients were similar to those of wild-type secondary recipients (Supplemental Fig. S6K-S), some $B u b 1 b^{H / H}$ secondary recipient mice had strong donor contribution ( $88 \%$ at $12 \mathrm{wk} ; n=6)$, one recipient mouse had weak donor contribution $(18.2 \%$ at $12 \mathrm{wk})$, and some recipient mice had an intermediate level of donor contribution ( $70 \%$ at $12 \mathrm{wk} ; n=3)$. Peripheral blood analyses revealed that the blood of the animal with a weak contribution of $B u b 1 b^{H / H}$ HSCs was largely comprised of CD45.1 cells, presumably descendants from rare recipient HSCs that survived irradiation (Fig. 5C). These data indicate that $B u b 1 b^{H / H}$ BM-HSCs lose their regenerative potential with serial transfer. To test this further, we performed a tertiary transfer with bone marrow cells derived from secondary recipients with high, intermediate, and low peripheral blood contribution from $B u b 1 b^{H / H}$ HSCs. Despite similar total HSC levels in the bone marrow of long-term reconstituted $B u b 1 b^{H / H}$ and wild-type secondary recipients (Supplemental Fig. S6T), mice that received bone marrow from secondary recipient donors with high $B u b 1 b^{H / H}$ peripheral blood contribution did not survive the tertiary transfer $(0 \%$ survival after $4 \mathrm{wk}$; $n=10)$. Mice that received bone marrow from donors with intermediate or low $B u b 1 b^{H / H}$ peripheral blood

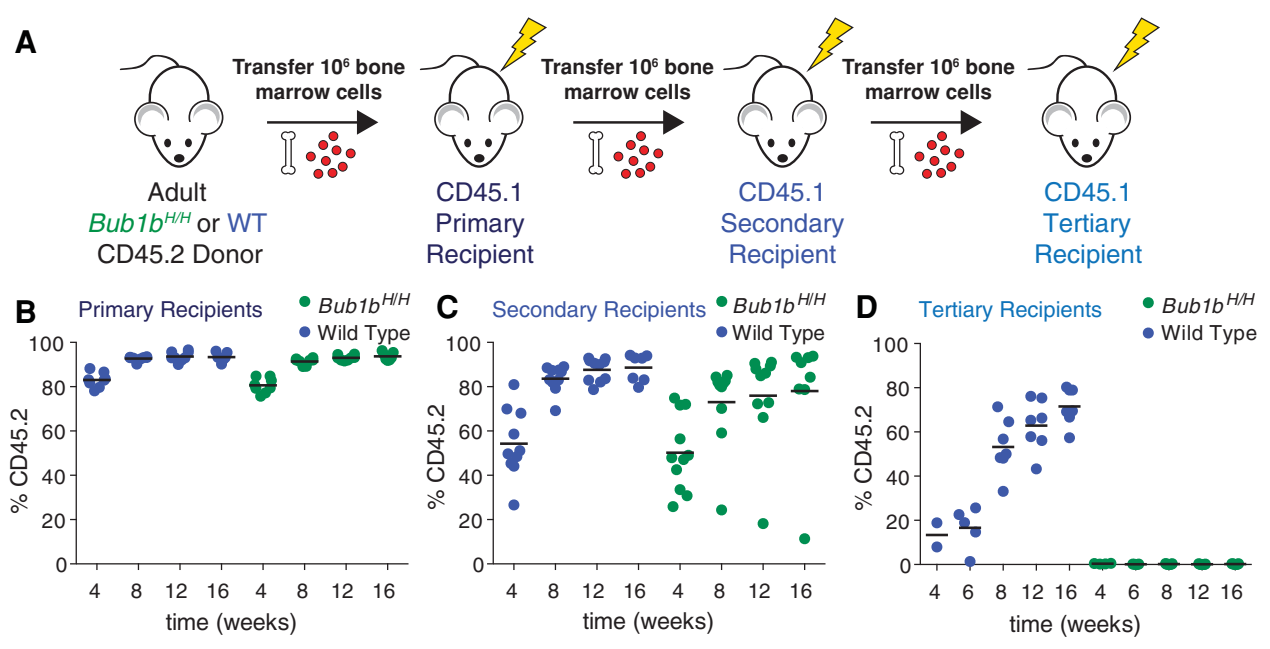

Figure 5. $B u b 1 b^{H / H}$ BM-HSCs undergo stem cell exhaustion upon serial reconstitution. (A) Schematic of serial reconstitution experiments. $(B-D)$ CD 45.2 adult $B u b 1 b^{H / H}$ bone marrow cells were serially transplanted into lethally irradiated CD45.1 recipients. The percentage of the peripheral blood that is CD45.2-positive was determined in primary $(B)$, secondary $(C)$, and tertiary $(D)$ recipients at the indicated times. The bar represents the mean value for each condition. 
contribution showed increased survival $160 \%$ and $80 \%$, respectively, after $16 \mathrm{wk} ; n=5$ for each condition). However, peripheral blood analyses of all mice that survived tertiary transfer revealed that virtually no cells originated from the original CD45.2 Bub1 $b^{H / H}$ donor; rather, cells were derived from some surviving CD45.1 HSCs from the recipients (Fig. 5D). This is in contrast to the tertiary recipients that received bone marrow from secondary recipients reconstituted with wild-type bone marrow, which, on average, had a $71.6 \%$ contribution from the wild-type donor after 16 wk $(n=7)$ (Fig. 5D). Thus, $B u b 1 b^{H / H}$ BM-HSCs lose the potential to reconstitute hematopoiesis upon serial transfer.

\section{Bub $1 b^{\mathrm{H} / \mathrm{H}}$ blood cells become progressively less aneuploid during hematopoietic reconstitution}

Initial characterization of the $B u b 1 b^{H / H}$ mouse model hypothesized that the aging-associated phenotypes observed in this mouse were due to progressive accumulation of aneuploid cells in adult tissues (Baker et al. 2004). Thus, $B u b 1 b^{H / H}$ BM-HSCs could be losing the potential to reconstitute the hematopoietic system upon serial transfer because the $B u b 1 b^{H / H}$ HSC pool becomes progressively more aneuploid with each successive proliferative challenge such that the level of aneuploidy in $B u b 1 b^{H / H}$ HSCs is too high to support effective proliferation. To test this possibility, we determined the karyotype of donor-derived peripheral white blood cells from one $B u b 1 b^{H / H}$ secondary recipient by single-cell sequencing (57 wk after transfer; $72 \%$ CD45.2 at time of sequencing). Surprisingly, all 17 cells sequenced were euploid (Fig. 6A, black triangle; Supplemental Fig. S7B). Thus, the failure of $B u b 1 b^{H / H} \mathrm{BM}-H S C$ s to reconstitute a tertiary recipient was not simply due to increased aneuploidy in blood cells derived from $B u b 1 b^{H / H}$ HSCs. Rather, the absence of aneuploid descendants of $B u b 1 b^{H / H}$ BM-HSCs suggests that aneuploid cells derived from these HSCs cannot effectively contribute to the peripheral blood of secondary recipients and that aneuploid cells are selected against. This inability of $B u b 1 b^{H / H}$ aneuploid BM-HSCs to contribute to the peripheral blood makes them unable to reconstitute tertiary recipients.

The lack of aneuploidy observed in the peripheral blood of a secondary recipient mouse of $B u b 1 b^{H / H}$ BMHSCs is in contrast to what we observed when we sequenced peripheral blood cells of a recipient mouse from a competitive reconstitution assay in which $38.9 \%$ of $B u b 1 b^{H / H}$ FL-HSC-derived peripheral blood cells or $19.45 \%$ of the total peripheral blood was aneuploid (Figs. 1G, 6A; Supplemental Fig. S7A, gray triangle). We hypothesized that differences in either the source of the HSCs (fetal liver or adult bone marrow) or the time of sampling could be responsible for the different levels of aneuploidy observed in the two analyses. Single-cell sequencing of $B u b 1 b^{H / H}$ FL-HSCs and BM-HSCs revealed that both of these cell types do not harbor any aneuploidies ( $n=19$ cells each) (Supplemental Fig. S7C,D), suggesting that the latter possibility was more likely responsible for the differences in aneuploidy observed.
The sample from the competitive reconstitution assay that revealed high levels of aneuploidy was obtained 16 wk after transfer of fetal liver donor cells, at a time during the reconstitution process when long-term hematopoiesis is just being established after a period a rapid expansion (Purton and Scadden 2007). In contrast, the sample from the secondary bone marrow recipient mouse that harbored euploid peripheral blood was obtained about a year after transfer of donor cells, when the bone marrow more closely resembled the steady state observed in an adult mouse.

The hypothesis that time after HSC transplantation can affect the degree of aneuploidy in the peripheral blood of recipient mice was informed by an evolutionary principle known as the "population flush" effect (Carson 1968). This principle states that, in rapidly expanding populations (as occurs immediately following transfer of donor HSCs to a lethally irradiated recipient), purifying selection is relaxed such that less-fit individuals can survive and significantly contribute to the population. A prediction of this hypothesis is that aneuploid $B u b 1 b^{H / H}$ peripheral blood cells would be more readily observed earlier during hematopoietic reconstitution, when the donor HSCs are rapidly proliferating to establish stable, longterm hematopoiesis, and less-fit cells-such as those generated by random chromosome missegregation in the $B u b 1 b^{H / H}$ adult mouse-would be tolerated. However, when populations reach steady state once long-term hematopoiesis has been established (as at 57 wk after secondary transplantation), purifying selection forces become relatively stronger, selecting against less-fit aneuploid cells. The observation that BM-HSCs are not aneuploid $(0 \%$ aneuploidy) is consistent with this principle. BMHSCs divide rarely, relying more on the division of progenitor cells to produce blood cells (Busch et al. 2015; Sun et al. 2015). Thus, in steady state, BM-HSCs likely experience strong purifying selection. FL-HSCs proliferate at E14.5, but this cell population remains relatively small even after expansion (Morrison et al. 1995; Ema and Nakauchi 2000). Additionally, FL-HSCs must still home to their niches before adult hematopoiesis commences (Orkin and Zon 2008), making it likely that this stem cell population is also under relatively strong purifying selection.

To directly test whether the population flush hypothesis can explain the degrees of aneuploidy observed in hematopoietic reconstitutions, we performed a time course, transferring $B u b 1 b^{H / H}$ fetal liver or bone marrow cells into lethally irradiated recipient mice and performing single-cell sequencing of peripheral blood cells 3, 6, 13, and $36 \mathrm{wk}$ after transfer to determine the levels of autosomal aneuploidy at these times. Single-cell sequencing of $B u b 1 b^{H / H}$ FL-HSCs and BM-HSCs, where no aneuploidy was observed, was used as the baseline level of aneuploidy in each population (0 time point in Fig. 6A; Supplemental Fig. S7C,D). In $B u b 1 b^{H / H}$ FL-HSC recipients, we found $8.3 \%$ of peripheral blood cells to be aneuploid 3 wk after transplantation ( $n=12$ cells) (Fig. 6A; Supplemental Fig. S8A). This percentage increased to $24 \%$ aneuploid cells 6 wk after transplantation $(n=25$ cells) (Fig. 6A; 

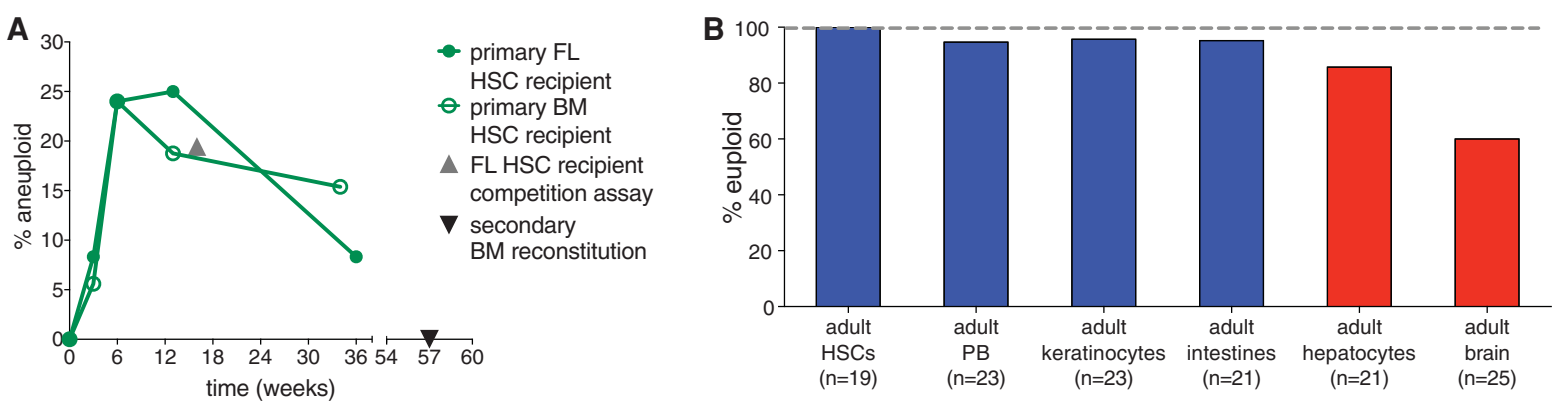

C

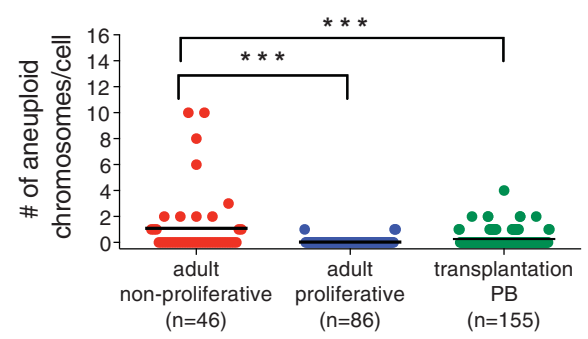

E

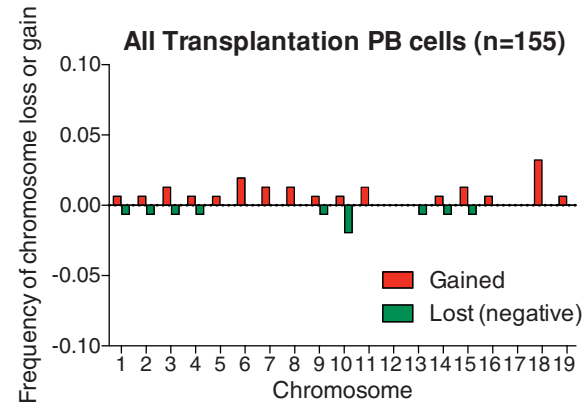

D

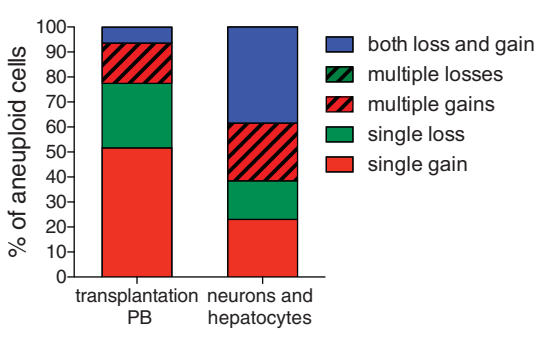

$\mathbf{F}$

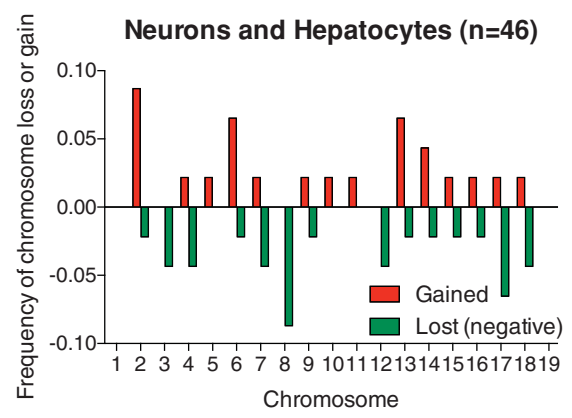

Figure 6. $B u b 1 b^{H / H}$ adult regenerative tissues show evidence of selection against aneuploid cells. $(A)$ The percent aneuploidy over time during hematopoietic reconstitution with $B u b 1 b^{H / H}$ HSCs was determined by single-cell sequencing of peripheral blood cells derived from primary recipient mice of $B u b 1 b^{H / H}$ bone marrow (open circles) or $B u b 1 b^{H / H}$ fetal liver (closed circles) cells at the indicated times after transfer. Peripheral blood cells from a mouse reconstituted with $B u b 1 b^{H / H}$ FL-HSCs 16 wk after competitive reconstitution (shown as the percent of total peripheral blood; gray triangle) (Fig. $1 \mathrm{G}$ ) and from a Bub1 $b^{H / H}$ secondary bone marrow recipient mouse (black triangle) (Fig. 5C) were also sequenced. Baseline aneuploidy was determined by single-cell sequencing of FL-HSCs and BM-HSCs. Segmentation plots of all sequenced cells are shown in Supplemental Figures S7 and S8. (B) Percentage of euploid cells found in different adult $B u b 1 b^{H / H}$ cell types. BM-HSCs, peripheral blood cells (PB), keratinocytes, and intestines (in blue) are from $\sim 4-$ mo-old $B u b 1 b^{H / H}$ mice. Data from hepatocytes and brains (in red) are from Knouse et al. (2014) and $\sim 6$-mo-old Bub1b ${ }^{H / H}$ mice. Segmentation plots of all newly sequenced cells are shown in Supplemental Figure S9. (C) The number of aneuploid chromosomes per cell in all adult cells analyzed in $A$ and $B$. The bar represents the mean value for each population. $t$-tests were performed for significance. $\left({ }^{* * *}\right) P<0.001$. $(D)$ Summary of chromosome gain and loss events observed in each cell from transplantation peripheral blood cells and adult nonproliferative neurons and hepatocytes. "Multiple gains" and "multiple losses" describe cells that have gained or lost two or more chromosomes. "Both loss and gain" describes cells that have gained at least one chromosome and lost at least one chromosome. $(E, F)$ Frequency of chromosome gain (red) or chromosome loss (green) by chromosome observed in all peripheral blood cells after transplantation $(E)$ and in all adult neurons and hepatocytes $(F)$.

Supplemental Fig. S8B). At $13 \mathrm{wk}, 25 \%$ of peripheral blood cells were aneuploid $(n=16$ cells) (Fig. 6A; Supplemental Fig. S8C). However, 36 wk after transplantation, the proportion of aneuploid peripheral blood cells dropped to $8.3 \%(n=12$ cells) (Fig. 6A; Supplemental Fig. S8D). Similar results were obtained in $B u b 1 b^{H / H}$ BM-HSC recipients. We found $5.6 \%$ of peripheral blood cells to be aneuploid $3 \mathrm{wk}$ after transplantation ( $n=18$ cells) (Fig. 6A; Supplemental Fig. S8E). At 6 wk, 24\% of peripheral blood cells were aneuploid ( $n=25$ cells) (Fig. 6A; Supplemental Fig. S8F), and, at $13 \mathrm{wk}, 18.8 \%$ of peripheral blood cells were aneuploid ( $n=16$ cells) (Fig. 6A; Supplemental
Fig. S8G). However, 34 wk after transplantation, the proportion of aneuploid peripheral blood cells dropped to $15.4 \%(n=13)$ (Fig. 6A; Supplemental Fig. S8H).

Taken together, our findings indicate that a higher level of aneuploidy is tolerated in the peripheral blood of $B u b 1 b^{H / H}$ recipient mice when the HSC pool is still expanding to establish long-term hematopoiesis after irradiation; however, these aneuploid cells are depleted from the peripheral blood of recipients once the HSC population is no longer rapidly expanding and the hematopoietic compartment reaches a steady state. Furthermore, we conclude that HSC source, either fetal liver or adult 
bone marrow, does not affect the degree of aneuploidy observed in the peripheral blood.

\section{Aneuploidy is selected against in $\mathrm{Bub} 1 \mathrm{~b}^{\mathrm{H} / \mathrm{H}}$ regenerating tissues}

The idea that rapidly expanding cell populations are more tolerant of aneuploidy than tissues that have reached a proliferative steady state further predicts that the prevalence of aneuploidy in adult $B u b 1 b^{H / H}$ tissues should depend on the proliferative capacity of tissues. Tissues that form during embryogenesis (when cells are rapidly proliferating) and are largely nonproliferative in the adult should harbor higher levels of aneuploidy. In contrast, tissues that undergo self-renewal and are thus under more stringent purifying selection in the adult should harbor lower levels of aneuploidy. To test this prediction, we sequenced single cells from tissues that self-renew in adult $B u b 1 b^{H / H}$ mice (peripheral blood cells, keratinocytes, and intestinal crypt cells) and compared the observed degree of autosomal aneuploidy with that of tissues that divide primarily during embryogenesis (the liver and brain). The liver and brain are formed during embryogenesis, and hepatocytes and neurons are largely nonproliferative in the adult (Zimmermann 2004; Campisi and d'Adda di Fagagna 2007). Our previous studies showed that aneuploid cells were prevalent in the livers $(18.8 \%)$ and brains $(38.1 \%)$ of 8 - and 12 -wk-old $B u b 1 b^{H / H}$ animals, respectively (Knouse et al. 2014). Sequencing four additional hepatocytes from a 30 -wk-old $B u b 1 b^{H / H}$ mouse and four additional neurons from a 28 -wk-old $B u b 1 b^{H / H}$ mouse estimated the levels of aneuploidy to be $14.3 \%$ and $40 \%$, respectively (Fig. 6B; Supplemental Fig. S9D,E).

Both BM-HSCs and peripheral white blood cells obtained from the same 19 -wk-old $B u b 1 b^{H / H}$ mouse exhibited $0 \%$ and $4.3 \%$ aneuploidy, respectively $(n=19$ and 23 cells sequenced) (Fig. 6B; Supplemental Figs. S7D, S9A). A previous study reported higher levels of aneuploidy in splenocytes of 3- and 5-mo-old $B u b 1 b^{H / H}$ animals (9\% and 15\%) (Baker et al. 2004). However, metaphase spreads, which tend to overestimate aneuploidy (Knouse et al. 2014), were used to evaluate cell karyotype in this study. It is also possible that aneuploid blood cells have a higher survival rate in the spleen than in the bone marrow or peripheral blood. Keratinocytes and intestinal cells enriched for crypts also showed low levels of aneuploidy: $4.3 \%$ of keratinocytes isolated from a 15 -wk-old $B u b 1 b^{H / H}$ mouse were aneuploid ( $n=23$ cells sequenced) (Fig. 6B; Supplemental Fig. S9B), and 4.8\% aneuploidy was observed in intestinal crypt cells from an 18-wk-old mouse and a 30 -wk-old $B u b 1 b^{H / H}$ mouse $(n=18$ and 3 cells sequenced, respectively) (Fig. 6B; Supplemental Fig. S9C). We conclude that tissues that regenerate during adulthood harbor lower levels of aneuploidy than tissues that proliferate predominantly during embryogenesis.

A direct comparison of the degree of aneuploidy between adult tissues further revealed that adult nonproliferative tissues (the brain and the liver) are more tolerant of aneuploidy than self-renewing adult tissues (peripheral blood, BM-HSCs, skin, and intestines) and blood cells derived from fetal liver or bone marrow transfers (Fig. 6C). Further analyses of specific chromosome gains and losses revealed that nonproliferative tissues (the brain and the liver) harbored cells with more complex aneuploidies than cells from $B u b 1 b^{H / H}$ HSC recipients (Fig. 6D-F; Supplemental Fig. S10). Of the three aneuploid cells observed in tissues that regenerate in the adult, two harbored a single chromosome gain, and one harbored a single chromosome loss (Supplemental Fig. S9). Thus, we conclude that aneuploidy is selected against in tissues that regenerate in the adult.

\section{Discussion}

Here we describe a system that has permitted direct comparison of aneuploid mammalian cell fitness with the fitness of isogenic euploid cells in vivo. We found that constitutional trisomy and aneuploidy resulting from CIN negatively affect HSC fitness. Furthermore, we obtained evidence that aneuploidy is selected against in adult regenerating tissues. These findings greatly inform our understanding of the role of aneuploidy in cancer and aging.

\section{The effects of aneuploidy and CIN on HSC fitness and hematopoiesis}

The hematopoietic reconstitution system that we developed here has allowed us to investigate whether aneuploidy provides a proliferative advantage to cells. Our findings demonstrate that both constitutional trisomy and aneuploidy generated by CIN adversely affect the fitness of HSCs in vivo. Defects specific to certain aneuploidies as well as defects observed in all aneuploid strains analyzed were evident in our aneuploid HSCs. The lineage-specific defects such as reduced B-cell number observed in trisomy 16 FL-HSC reconstitutions are likely due to chromosome-specific effects, as B-cell differentiation defects have also been observed in DS (Roy et al. 2012; Lane et al. 2014). Furthermore, we observed decreased proliferative potential in all aneuploidy models that we examined. Trisomy 16 FL-HSCs are much less fit than euploid HSCs and show phenotypes characteristic of proliferation defects when challenged to reconstitute irradiated euploid recipients. Trisomy 19 and $B u b 1 b^{H / H}$ HSCs exhibit less severe fitness defects, indicating that lower levels of aneuploidy are better tolerated in the hematopoietic lineage. Recent studies have shown that murine HSCs divide very infrequently and use numerous, more differentiated progenitor cells to sustain long-term hematopoiesis (Busch et al. 2015; Sun et al. 2015). This provides a potential explanation of why repeated challenges are needed to reveal the fitness defects of aneuploidy in $B u b 1 b^{H / H}$ HSCs. In the $B u b 1 b^{H / H}$ HSC pool, only a subpopulation of cells is likely aneuploid, and selection for euploid cells likely occurs given that we did not observe aneuploidy in $B u b 1 b^{H / H}$ HSCs. In the same vein, a proliferative defect in trisomy 19 HSCs began to emerge after tertiary bone marrow transfer and was 
obvious only after quaternary transfer. Thus, in vivo analyses of primary aneuploid cells demonstrate that aneuploidy in the hematopoietic lineage reduces cellular fitness and proliferative capacity. While some aneuploidies, such as trisomy 19, are better tolerated than others, such as trisomy 16, reconstitutions with the $B u b 1 b^{H / H}$ CIN model reveal that when euploid cells can be generated, aneuploidy is selected against in the hematopoietic lineage.

How aneuploidy leads to reduced fitness in HSCs remains to be determined. Cell culture studies of yeast and mammalian cells have shown that the proteomic imbalances caused by aneuploidy lead to proteotoxic stress, metabolic alterations, increased reactive oxygen species (ROS) production, and cell cycle delays (Santaguida and Amon 2015). These general characteristics of aneuploid cells likely contribute to the decreased proliferative potential and eventual exhaustion of aneuploid HSCs that are observed.

While increased proliferation was not observed in transplantation of aneuploid HSCs alone, it will be interesting to determine whether particular aneuploidies can contribute to tumorigenesis in specific oncogenic contexts or in conjunction with aneuploidy-tolerating mutations. For example, aneuploidy has been shown to increase tumor burden and decrease survival in animals lacking the tumor suppressor p53 (Li et al. 2010). Loss of p53 could permit the survival of cells with abnormal karyotypes and/or DNA damage that ensues when chromosomes are missegregated (Crasta et al. 2012), allowing for the selection of malignant karyotypes. Particular aneuploidies in conjunction with other specific genomic alterations could also facilitate the development of specific cancers. For example, analyses of individuals with DS demonstrate that trisomy 21 is sufficient to bias differentiation in the human fetal liver, leading to a relative expansion of myeloid cells (Chou et al. 2008; TunstallPedoe et al. 2008). This bias can progress to a transient myeloproliferative disorder (TMD) (Gamis and Smith 2012) and eventually AML if a cooperating GATA1 mutation is also present (Hitzler 2003; Mundschau et al. 2003). Furthermore, trisomy 21 is also observed in non-DS hematologic cancers (Mitelman et al. 1990; Cheng et al. 2009). The model system that we developed here will permit effective molecular dissection of the effect of specific chromosomal abnormalities and the role of aneuploidy and CIN in general on the development of leukemias and lymphomas.

\section{Aneuploidy is selected against in regenerating tissues}

Our finding that aneuploidy impairs rather than promotes proliferation in vivo is supported by our tissue analyses in $B u b 1 b^{H / H}$ mice. Tissues that form largely during embryogenesis harbored high levels of aneuploid cells, whereas BM-HSCs and adult peripheral blood, skin, and intestinal crypt cells harbor few aneuploid cells. Importantly, we also found that FL-HSCs were euploid, suggesting that mechanisms that eliminate aneuploid cells are not unique to adult tissues. Rather, we favor the idea that the varying degree of aneuploidy observed in different tissues reflects differences in the strength of purifying selection across tissues. During rapid population expansion, a "population flush" effect (Carson 1968) can occur in which genetically less-fit individuals are able to survive and contribute to the population in a more substantive manner. Once populations reach steady state, purifying selection forces are relatively stronger and select against the survival of less-fit individuals. Thus, tissues that form during embryogenesis, a period of rapid cell expansion, and are largely nonproliferative and not maintained by stem cells in the adult (brain and liver) harbor high levels of aneuploidy, whereas adult regenerating tissues (blood, skin, and the intestine) harbor few aneuploid cells. Our analysis of aneuploidy dynamics in $B u b 1 b^{H / H}$ HSC-derived blood cells during hematopoietic reconstitution further indicates that the way in which tissues are generated and maintained determines the prevalence of aneuploidy in those tissues. Both BM-HSCs and FL-HSCs are euploid. However, when HSCs are challenged to rapidly expand to reconstitute an irradiated recipient, aneuploidy becomes more prevalent in the peripheral blood of primary recipients during the early stages of hematopoietic reconstitution. Aneuploidy levels then decline as the tissue reaches steady state.

A previous evaluation of aneuploidy in aged mice that harbored increased expression of the BUBR1 protein in all tissues throughout life found that cells that overexpress BUBR1 and additionally all wild-type aged adult stem cells show low levels of aneuploidy (Baker et al. 2012). We found that, even in a mutant in which the BUBR1 checkpoint protein is compromised, aneuploidy is selected against in tissues that are maintained by adult stem cells. Further investigation is needed to determine how aneuploid cells are culled in adult regenerating tissues. Previous cell culture studies have shown that chromosome missegregation leads to p53 activation (Li et al. 2010; Thompson and Compton 2010), raising the possibility that aneuploid cells are culled in tissues through apoptosis. We did not observe high levels of apoptosis in the skin or bone marrow of adult $B u b 1 b^{H / H}$ mice by standard assays (data not shown), suggesting that other mechanisms are responsible for eliminating aneuploid cells in adult regenerating tissues.

\section{Aneuploidy and aging}

$B u b 1 b^{H / H}$ mice have a decreased life span and show a number of progeroid phenotypes such as dwarfism, cataracts, loss of subcutaneous fat, and impaired wound healing (Baker et al. 2004). It has been proposed that these early aging phenotypes are caused by the progressive accumulation of aneuploid cells in these animals. However, our findings that serial bone marrow transfer leads to $B u b 1 b^{H / H}$ HSC exhaustion and that adult $B u b 1 b^{H / H}$ regenerating tissues contain very few aneuploid cells suggest an alternative explanation. The decreased life span and early aging phenotypes exhibited by $B u b 1 b^{H / H}$ mice could be due to the exhaustion of adult stem cell pools that must proliferate more than wild-type stem cells to 
produce euploid cells. It is also possible that the strength of purifying selection in adult regenerative tissues is relaxed as organisms age and stem cell fitness declines (Rozhok and DeGregori 2015). Such decreased purifying selection would cause tissue function decline due to the accumulation of aneuploid and hence less-fit cells with time. Consistent with this idea is the observation that life span extension can be achieved in $B u b 1 b^{H / H}$ mice when senescing, and thus presumably aneuploid cells are induced to undergo apoptosis (Baker et al. 2011). Further investigation is needed to determine whether decreased purifying selection can also cause the accumulation of cells that are genetically unstable and karyotypically abnormal (Sheltzer et al. 2011; Zhu et al. 2012; Nicholson et al. 2015), since such a population from which rare cells with high proliferative and hence tumorigenic potential may arise could cause cancer as individuals age.

\section{Materials and methods}

Mouse strains

The mouse strains used previously (Williams et al. 2008) were backcrossed for at least 10 generations into the C57BL/6J background (Jackson Laboratory) to generate congenic strains. Strains used to generate trisomic embryos were B6.Cg-Rb(6.16)24Lub/ JAmonJ or B6.Cg-Rb(13.16)1Mpl/JAmonJ and B6.Cg-Rb(16.17) 7Bnr/JAmonJ (trisomy 16) and B6.Cg-Rb(5.19)1Wh/JAmonJ and B6Ei.Cg-Rb(9.19)163H/J (trisomy 19). All male compound heterozygous mice were mated with C57BL/6J females to generate trisomic embryos, although at a lower frequency than previously reported, most likely due to backcrossing (Pfau and Amon 2016). Bub1b $b^{H / H}$ mice were a generous gift from Dr. J.M. van Deursen. Embryos from all strains were collected at E14.5E15.5 by timed matings. Recipient mice were 6- to 8-wk-old B6. SJL-Ptprc ${ }^{a}$ Pepc $^{b}$ /BoyJ (CD45.1) female mice from Jackson Laboratory. Fetal livers were prepared and karyotyped as described in the Supplemental Material. All animal studies and procedures were approved by the Massachusetts Institute of Technology Institutional Animal Care and Use Committee.

\section{Hematopoietic reconstitutions}

For all reconstitution experiments, mice were closely monitored for signs of bone marrow failure and rapid weight loss. All protocols for treating irradiated mice were outlined by the Massachusetts Institute of Technology's Division of Comparative Medicine. Whole-body irradiation was performed using a $137^{\mathrm{Cs}}$ irradiator ( $\gamma$ cell 40 ) at a dose rate of $\sim 100 \mathrm{cGy} / \mathrm{min}$.

For competitive reconstitution assays and hematopoietic reconstitution experiments, recipient mice were irradiated with a 12 Gy total dose administered as a split dose of $8 \mathrm{~Gy}$ followed by a second dose of $4 \mathrm{~Gy} 3 \mathrm{~h}$ later. Fetal liver cells were thawed in Iscove's modified Dulbecco's medium (IMDM) supplemented with 2\% FBS and counted on a Cellometer Auto T4 automated hemacytometer (Nexelcom). Viability was assessed by propidium iodide exclusion using a FACSCalibur flow cytometer (Becton Dickinson). Live cells ( $10^{6}$ cells) were injected intravenously in Hank's balanced salt solution (HBSS). In one replicate of the trisomy 16 competition assay ( $n=5$ trisomy 16; $n=7$ wild type), CD45.2/ CD45.1 aneuploid donors and CD45.2 common wild-type donors were transferred into CD45.1 irradiated recipient mice.
Recipient mice for CFU-S assays were irradiated with 9.5 Gy administered as a single dose. Fetal liver cells were then prepared, and $10^{6}$ live cells were injected as described above. Seven days to 8 $\mathrm{d}$ after injection, spleens were harvested from recipients and then fixed overnight in Bouin's fixative. Spleens were sectioned into 5$\mu \mathrm{m}$ slices and stained with hematoxylin and eosin. Slides were then scanned on a Leica Aperio slide scanner, and colony area was measured using ImageJ. To control for small residual white blood cell nodules in the spleen, sections of spleens from irradiated mice that were not transferred with cells were also analyzed. We determined the average background colony size to be $0.336 \%$ of the total spleen area, and colonies larger than this average were considered CFU-S colonies.

Homing assays were performed as described (Gilbert and Hemann 2012) and are detailed in the Supplemental Material.

For EdU incorporation assays, recipient mice were irradiated with $9.5 \mathrm{~Gy}$, administered as a single dose. Fetal liver cells were thawed, and viability was determined by propidium iodide exclusion as described above. Live fetal liver cells $\left(10^{6}\right.$ cells $)$ were injected intravenously in HBSS. Six days later, mice were injected intraperitoneally with $1.25 \mathrm{mg}$ of EdU in PBS. Twenty hours after EdU injection, recipient mice were euthanized. Bone marrow was harvested by flushing the long bones $24 \mathrm{~h}$ after injection. Red blood cells were lysed in ACK lysing buffer, and samples were washed in IMDM containing $2 \%$ FBS. EdU incorporation was detected using the Click-iT plus EdU Alexa fluor 488 flow cytometry kit (Thermo-Fisher) on an LSR II flow cytometer (Becton Dickinson). EdU-positive recipient-derived bone marrow cells were excluded using a CD45.1 antibody conjugated to PE (BioLegend; clone A20).

Recipient mice for bone marrow transfers were irradiated with $9.5 \mathrm{~Gy}$, administered as a single dose. Bone marrow cells were isolated, and red blood cells were lysed as described above. White blood cells were then counted, and recipient mice were reconstituted by intravenous injection of $10^{6}$ cells in HBSS.

\section{Peripheral blood analysis}

For complete blood cell counts, peripheral blood was collected with heparinized capillary tubes into EDTA-coated Microvette 100 tubes (Sarstedt) and analyzed on a HemaVet 950FS (Drew Scientific). Peripheral blood for flow cytometry analysis was collected with heparinized capillary tubes into sodium heparin diluted in PBS. Red blood cells were lysed in ACK lysing buffer and washed in HBSS containing $2 \%$ FBS. Cells were then incubated with antibodies according to the manufacturer's specifications and analyzed with a FACSCalibur or LSR II flow cytometer (Becton Dickinson). The following antibodies were used and obtained from BioLegend: CD45.1 (A20), CD45.2 (104), CD45R/B220 (RA3-6B2), and CD90.2/Thy-1.2 (53-2.1).

\section{HSC quantification}

Fetal livers and bone marrow cells were harvested, genotyped or karyotyped, processed, and counted as described above for hematopoietic reconstitutions. FL-HSCs were quantified using markers described previously (Kim et al. 2006). BM-HSCs were quantified as described previously (Kiel et al. 2005). More detailed procedures are included in the Supplemental Material. The following antibodies were used and obtained from BioLegend: CD150 (TC15-12F12.2), CD48 (HM48-1), Sca-1 (E13-161.7), CD117 (2B8). The following antibodies were used in a lineage cocktail for fetal liver cells and were obtained from BioLegend: CD3e (145-2C11), Ly-6G/Ly-6C (Gr-1) (RB6-8C5), B220/CD45R (RA3-6B2), Ter-119, CD5 (53-7.3), and CD8a (53-6.7). 


\section{Single-cell sequencing and trisomy 19 sequencing}

Single-cell sequencing was performed and analyzed as described in Knouse et al. (2014). Single-cell isolation protocols for each cell type are described in detail in the Supplemental Material. CD45.2 trisomy 19 peripheral blood was prepared as above for other blood cells and sorted on a FACSAria (Becton Dickinson). Genomic DNA was isolated from collected cells using the DNeasy blood and tissue kit (Qiagen), amplified with GenomePlex Complete WGA kit (Sigma-Aldrich), and sequenced and analyzed in the same way as amplified single cells. The sequences reported in this study have been deposited in the National Center for Biotechnology Information Sequence Read Archive (accession no. SRP075831).

\section{Acknowledgments}

We thank the Swanson Biotechnology Center at the Koch Institute for Integrative Cancer Research; Paul Danielian and Christian Pallasch for advice and technical assistance; the Massachusetts Institute of Technology Division of Comparative Medicine diagnostic laboratory for technical assistance; Jie Wu for help with bioinformatics analysis; Jan van Deursen for generously providing the Bub1b $b^{H / H}$ mouse strain; David Housman, Michael Hemann, James DeGregori, Andrii Rozhok, Jianzhu Chen, and Ömer Yilmaz for discussions and advice; and Jianzhu Chen, Ömer Yilmaz, and members of the Amon laboratory for critical reading of this manuscript. This work was supported by the National Institutes of Health (CA206157) to A.A., and the Kathy and Curt Marble Cancer Research Fund and in part by Koch Institute Support Grant P30-CA14051. S.J.P. is supported by a National Science Foundation Graduate Research Fellowship, and K.A.K. is supported by the National Institute of General Medical Sciences Training Grant T32GM007753. A.A. is an investigator of the Howard Hughes Medical Institute and the Glenn Foundation for Biomedical Research.

\section{References}

Adorno M, Sikandar S, Mitra SS, Kuo A, Di Robilant BN, HaroAcosta V, Ouadah Y, Quarta M, Rodriguez J, Qian D, et al. 2013. Usp16 contributes to somatic stem-cell defects in Down's syndrome. Nature 501: 380-384.

Babu J, Jeganathan K, Baker D, Wu X, Kang-Decker N, van Deursen J. 2003. Rael is an essential mitotic checkpoint regulator that cooperates with Bub3 to prevent chromosome missegregation. I Cell Biol 160: 341-353.

Baker DJ, Jeganathan KB, Cameron JD, Thompson M, Juneja S, Kopecka A, Kumar R, Jenkins RB, de Groen PC, Roche P, et al. 2004. BubR1 insufficiency causes early onset of agingassociated phenotypes and infertility in mice. Nat Genet 36: 744-749.

Baker DJ, Wijshake $T$, Tchkonia $\mathrm{T}$, LeBrasseur NK, Childs BG, van de Sluis B, Kirkland JL, van Deursen JM. 2011. Clearance of p16Ink4a-positive senescent cells delays ageing-associated disorders. Nature 479: 232-236.

Baker DJ, Dawlaty MM, Wijshake T, Jeganathan KB, Malureanu L, van Ree JH, Crespo-Diaz R, Reyes S, Seaburg L, Shapiro V, et al. 2012. Increased expression of BubR1 protects against aneuploidy and cancer and extends healthy lifespan. Nat Cell Biol 14: 1-9.

Boveri T. 1914. Zur frage der entstehung maligner tumoren. Gustav Fischer, Jena, Germany.

Busch K, Klapproth K, Barile M, Flossdorf M, Holland-Letz T, Schlenner SM, Reth M, Höfer T, Rodewald H-R. 2015. Funda- mental properties of unperturbed haematopoiesis from stem cells in vivo. Nature 518: 542-546.

Campisi J, d'Adda di Fagagna F. 2007. Cellular senescence: when bad things happen to good cells. Nat Rev Mol Cell Biol 8: 729-740.

Carson HL. 1968. The population flush and its genetic consequences. In Population biology and evolution (ed. Lewontin RC), pp. 123-137. Syracuse University Press, Syracuse, NY.

Cheng Y, Wang H, Wang H, Chen Z, Jin J. 2009. Trisomy 21 in patients with acute leukemia. Am J Hematol 84: 193-194.

Choi JK. 2008. Hematopoietic disorders in Down syndrome. Int $J$ Clin Exp Pathol 1: 387-395.

Chou ST, Opalinska JB, Yao Y, Fernandes MA, Kalota A, Brooks JSJ, Choi JK, Gewirtz AM, Danet-Desnoyers G-A, Nemiroff RL, et al. 2008. Trisomy 21 enhances human fetal erythromegakaryocytic development. Blood 112: 4503-4506.

Crasta K, Ganem NJ, Dagher R, Lantermann AB, Ivanova EV, Pan Y, Nezi L, Protopopov A, Chowdhury D, Pellman D. 2012. DNA breaks and chromosome pulverization from errors in mitosis. Nature 482: 53-58.

Davoli T, Xu AW, Mengwasser KE, Sack LM, Yoon JC, Park PJ, Elledge SJ. 2013. Cumulative haploinsufficiency and triplosensitivity drive aneuploidy patterns and shape the cancer genome. Cell 155: 948-962.

Ema H, Nakauchi H. 2000. Expansion of hematopoietic stem cells in the developing liver of a mouse embryo. Blood 95: 2284-2288.

Frasca D, Guidi F, Arbitrio M, Pioli C, Poccia F, Cicconi R, Doria G. 2000. Hematopoietic reconstitution after lethal irradiation and bone marrow transplantation: effects of different hematopoietic cytokines on the recovery of thymus, spleen and blood cells. Bone Marrow Transplant 25: 427-433.

Gamis AS, Smith FO. 2012. Transient myeloproliferative disorder in children with Down syndrome: clarity to this enigmatic disorder. Br J Haematol 159: 277-287.

Gilbert LA, Hemann MT. 2012. Context-specific roles for paracrine IL-6 in lymphomagenesis. Genes Dev 26: 1758-1768.

Gogendeau D, Siudeja K, Gambarotto D, Pennetier C, Bardin AJ, Basto R. 2015. Aneuploidy causes premature differentiation of neural and intestinal stem cells. Nat Commun 6: 8894.

Gropp A, Winking H, Herbst EW, Claussen CP. 1983. Murine trisomy: developmental profiles of the embryo, and isolation of trisomic cellular systems. J Exp Zool 228: 253-269.

Harrison DE, Astle CM. 1982. Loss of stem cell repopulating ability upon transplantation. Effects of donor age, cell number, and transplantation procedure. J Exp Med 156: 1767-1779.

Harrison DE, Zhong RK, Jordan CT, Lemischka IR, Astle CM. 1997. Relative to adult marrow, fetal liver repopulates nearly five times more effectively long-term than short-term. Exp Hematol 25: 293-297.

Henry E, Walker D, Wiedmeier SE, Christensen RD. 2007. Hematological abnormalities during the first week of life among neonates with Down syndrome: data from a multihospital healthcare system. Am J Med Genet 143A: 42-50.

Herbst EW, Winking H. 1991. Adoptive transfer of the hematopoietic system of trisomic mice with limited life span: stem cells from six different trisomies are capable of survival. $\mathrm{Dev}$ Genet 12: 415-422.

Hitzler JK. 2003. GATA1 mutations in transient leukemia and acute megakaryoblastic leukemia of Down syndrome. Blood 101: 4301-4304.

Jeganathan K, Malureanu L, Baker DJ, Abraham SC, van Deursen JM. 2007. Bub1 mediates cell death in response to 
chromosome missegregation and acts to suppress spontaneous tumorigenesis. J Cell Biol 179: 255-267.

Kiel MJ, Yilmaz OH, Iwashita T, Yilmaz OH, Terhorst C, Morrison SJ. 2005. SLAM family receptors distinguish hematopoietic stem and progenitor cells and reveal endothelial niches for stem cells. Cell 121: 1109-1121.

Kiladjian J-J. 2012. The spectrum of JAK2-positive myeloproliferative neoplasms. Hematology 2012: 561-566.

Kim I, He S, Yilmaz OH, Kiel MJ, Morrison SJ. 2006. Enhanced purification of fetal liver hematopoietic stem cells using SLAM family receptors. Blood 108: 737-744.

Knouse KA, Wu J, Whittaker CA, Amon A. 2014. Single cell sequencing reveals low levels of aneuploidy across mammalian tissues. Proc Nat1 Acad Sci 111: 13409-13414.

Kondo M, Wagers AJ, Manz MG, Prohaska SS, Scherer DC, Beilhack GF, Shizuru JA, Weissman IL. 2003. Biology of hematopoietic stem cells and progenitors: implications for clinical application. Annu Rev Immunol 21: 759-806.

Lane AA, Chapuy B, Lin CY, Tivey T, Li H, Townsend EC, van Bodegom D, Day TA, Wu S-C, Liu H, et al. 2014. Triplication of a $21 \mathrm{q} 22$ region contributes to $\mathrm{B}$ cell transformation through HMGN1 overexpression and loss of histone H3 Lys27 trimethylation. Nat Genet 46: 618-623.

Lapidot T. 2005. How do stem cells find their way home? Blood 106: 1901-1910.

Li M, Fang X, Baker DI, Guo L, Gao X, Wei Z, Han S, van Deursen JM, Zhang P. 2010. The ATM-p53 pathway suppresses aneuploidy-induced tumorigenesis. Proc Natl Acad Sci 107: 14188-14193.

Mitelman F, Heim S, Mandahl N. 1990. Trisomy-21 in neoplastic cells. Am J Med Genet 7: 262-266.

Morrison SJ, Hemmati HD, Wandycz AM, Weissman IL. 1995. The purification and characterization of fetal liver hematopoietic stem cells. Proc Natl Acad Sci 92: 10302-10306.

Mundschau G, Gurbuxani S, Gamis AS, Greene ME, Arceci RJ, Crispino JD. 2003. Mutagenesis of GATA1 is an initiating event in Down syndrome leukemogenesis. Blood 101: 42984300.

Nicholson JM, Macedo JC, Mattingly AJ, Wangsa D, Camps J, Lima V, Gomes AM, Dória S, Ried T, Logarinho E, et al. 2015. Chromosome mis-segregation and cytokinesis failure in trisomic human cells. eLife 4: e05068.

Orkin SH, Zon LI. 2008. Hematopoiesis: an evolving paradigm for stem cell biology. Cell 132: 631-644.

Pfau SI, Amon AA. 2012. Chromosomal instability and aneuploidy in cancer: from yeast to man. EMBO Rep 13: 515-527.

Pfau SJ, Amon A. 2016. A system to study aneuploidy in vivo. Cold Spring Harb Symp Quant Biol 80: 027193.

Purton LE, Scadden DT. 2007. Limiting factors in murine hematopoietic stem cell assays. Cell Stem Cell 1: 263-270.

Rajagopalan H, Lengauer C. 2004. Aneuploidy and cancer. Nature 432: 338-341.
Roy A, Cowan G, Mead AJ, Filippi S, Bohn G, Chaidos A, Tunstall O, Chan JKY, Choolani M, Bennett P, et al. 2012. Perturbation of fetal liver hematopoietic stem and progenitor cell development by trisomy 21. Proc Natl Acad Sci 109: 17579-17584.

Rozhok AI, DeGregori J. 2015. Toward an evolutionary model of cancer: considering the mechanisms that govern the fate of somatic mutations. Proc Natl Acad Sci 112: 8914-8921.

Santaguida S, Amon A. 2015. Short- and long-term effects of chromosome mis-segregation and aneuploidy. Nat Rev Mol Cell Biol 16: 473-485.

Satge D, Sommelet D, Geneix A, Nishi M, Malet P, Vekemans M. 1998. A tumor profile in Down syndrome. Am J Med Genet 78: 207-216.

Segal DI, McCoy EE. 1974. Studies on Down's syndrome in tissue culture. I. Growth rates protein contents of fibroblast cultures. J Cell Physiol 83: 85-90.

Sheltzer JM, Blank HM, Pfau SJ, Tange Y, George BM, Humpton TJ, Brito IL, Hiraoka Y, Niwa O, Amon A. 2011. Aneuploidy drives genomic instability in yeast. Science 333: 1026-1030.

Stingele S, Stoehr G, Peplowska K, Cox JUR, Mann M, Storchova Z. 2012. Global analysis of genome, transcriptome and proteome reveals the response to aneuploidy in human cells. Mol Syst Biol 8: 608.

Sun J, Ramos A, Chapman B, Johnnidis JB, Le L, Ho Y-J, Klein A, Hofmann O, Camargo FD. 2015. Clonal dynamics of native haematopoiesis. Nature 514: 322-327.

Thompson SL, Compton DA. 2010. Proliferation of aneuploid human cells is limited by a p53-dependent mechanism. I Cell Biol 188: 369-381.

Till JE, McCulloch EA. 1961. A direct measurement of the radiation sensitivity of normal mouse bone marrow cells. Radiat Res 14: 213-222.

Tunstall-Pedoe O, Roy A, Karadimitris A, de la Fuente J, Fisk NM, Bennett P, Norton A, Vyas P, Roberts I. 2008. Abnormalities in the myeloid progenitor compartment in Down syndrome fetal liver precede acquisition of GATA1 mutations. Blood 112: 4507-4511.

Waterstrat A, Liang Y, Swiderski CF, Shelton BJ, Van Zant G. 2010. Congenic interval of CD45/Ly-5 congenic mice contains multiple genes that may influence hematopoietic stem cell engraftment. Blood 115: 408-417.

Weaver BAA, Silk AD, Montagna C, Verdier-Pinard P, Cleveland DW. 2007. Aneuploidy acts both oncogenically and as a tumor suppressor. Cancer Cell 11: 25-36.

Williams BR, Prabhu VR, Hunter KE, Glazier CM, Whittaker CA, Housman DE, Amon A. 2008. Aneuploidy affects proliferation and spontaneous immortalization in mammalian cells. Science 322: 703-709.

Zhu J, Pavelka N, Bradford WD, Rancati G, Li R. 2012. Karyotypic determinants of chromosome instability in aneuploid budding yeast. PLoS Genet 8: e1002719.

Zimmermann A. 2004. Regulation of liver regeneration. Nephrol Dial Transplant 19: iv6-i10. 


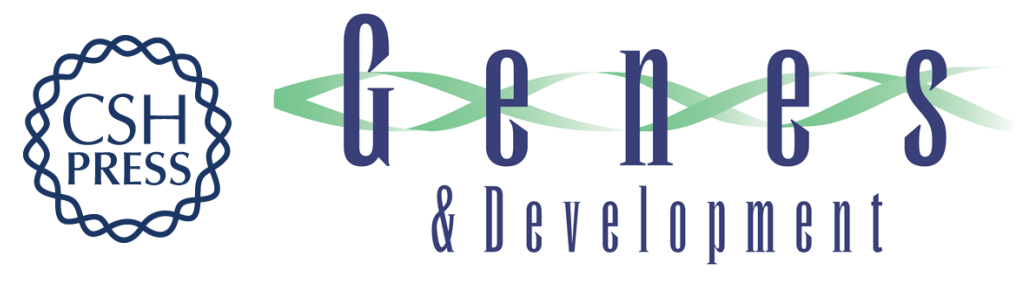

\title{
Aneuploidy impairs hematopoietic stem cell fitness and is selected against in regenerating tissues in vivo
}

\author{
Sarah J. Pfau, Rebecca E. Silberman, Kristin A. Knouse, et al.
}

Genes Dev. 2016, 30: originally published online June 16, 2016

Access the most recent version at doi:10.1101/gad.278820.116

\section{Supplemental http://genesdev.cshlp.org/content/suppl/2016/06/16/gad.278820.116.DC1 Material}

References This article cites 57 articles, 22 of which can be accessed free at: http://genesdev.cshlp.org/content/30/12/1395.full.html\#ref-list-1

Creative This article is distributed exclusively by Cold Spring Harbor Laboratory Press for the first Commons six months after the full-issue publication date (see

License http://genesdev.cshlp.org/site/misc/terms.xhtml). After six months, it is available under a Creative Commons License (Attribution-NonCommercial 4.0 International), as described at http://creativecommons.org/licenses/by-nc/4.0/.

Email Alerting Receive free email alerts when new articles cite this article - sign up in the box at the top Service right corner of the article or click here.

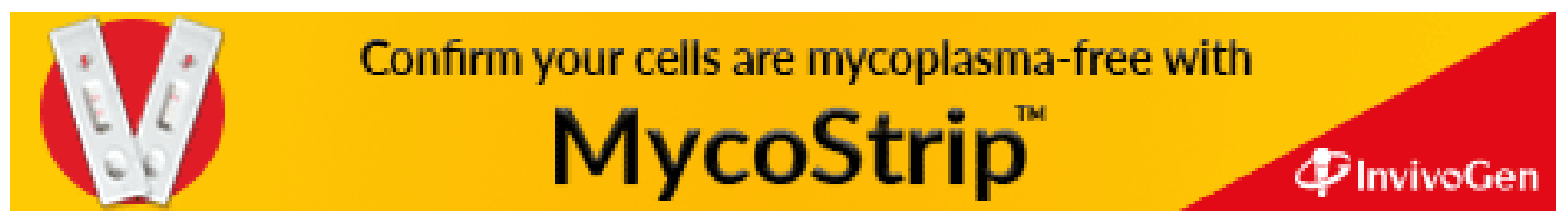

